\title{
Prefrontal Cortex Long-Term Potentiation, But Not Long-Term Depression, Is Associated with the Maintenance of Extinction of Learned Fear in Mice
}

\author{
Cyril Herry ${ }^{1}$ and René Garcia ${ }^{2}$ \\ 1 Laboratoire de Neurosciences Cognitives, Centre National de la Recherche Scientifique Unité Mixte de Recherche 5106, \\ Université de Bordeaux I, 33405 Talence, France, and 2Laboratoire de Psychophysiologie, Faculté des Sciences, \\ Université de Nice-Sophia Antipolis, 06108 Nice, France
}

Considerable efforts have been made to identify changes of brain synaptic plasticity associated with fear conditioning. However, for both clinical applications and our fundamental understanding of memory processes, it appears also necessary to investigate synaptic plasticity related to extinction. We previously showed that extinction of freezing to a tone conditioned stimulus (CS; previously paired with footshock) in mice results in a sequence of depression and potentiation of synaptic efficacy in the medial prefrontal cortex (mPFC). These data as well as those from lesion studies suggest that the direction of changes in prefrontal synaptic plasticity may modulate extinction of learned fear. To test this, we analyzed the effects of low-frequency stimulation (LFS) and high-frequency stimulation (HFS) of the mediodorsal thalamic nucleus, known to induce prefrontal long-term depression (LTD) and potentiation (LTP),

Exposure to traumatic events can precipitate long-lasting changes in affect (Ettedgui and Bridges, 1985; Friedman, 1997; Yehuda et al., 1998) without any manifestation of structural brain damage (Markowitsch et al., 1998), but with long-lasting changes of synaptic plasticity in certain brain structures (Garcia, 2001). Because affective changes can dissipate after cognitive therapy, which constitutes a form of extinction learning, further changes in synaptic plasticity (for example, reversal of the earlier plasticity) might also occur in these structures, to either sustain or promote long-term memory of treatment (extinction). Consequently, a failure to develop these changes in synaptic plasticity during or after the treatment may lead to chronic posttraumatic stress disorder (PTSD).

An examination of the changes in synaptic plasticity in the medial prefrontal cortex (mPFC) may be very informative in this context. First, affective changes related to PTSD are accompanied by both cognitive dysfunction (Markowitsch et al., 1998; Vasterling et al., 1998; Moradi et al., 1999) and a decrease in neuronal activity in this cortical region (Bremner et al., 1999a,b; Fernandez et al., 2001). Second, clinical follow-up data show that benefits from cognitive therapy, which require normal function-

Received Aug. 27, 2001; revised Oct. 17, 2001; accepted Oct. 25, 2001.

This work was supported by the Centre National de la Recherche Scientifique, the Conseil Régional d'Aquitaine, and a grant from the Fondation pour la Recherche Médicale to C.H.

Correspondence should be addressed to Dr. René Garcia, Laboratoire de Psychophysiologie, Faculté des Sciences, Université de Nice-Sophia Antipolis, Parc Valrose, 06108 Nice, France. E-mail: rene.garcia@unice.fr.

Copyright (ㄷ) 2002 Society for Neuroscience $0270-6474 / 02 / 220577-07 \$ 15.00 / 0$ respectively, on extinction. We found that maintenance of the depression phase, using thalamic LFS, was associated with resistance to extinction. Thalamic HFS applied before extinction testing had no effect on the rate of extinction. However, 1 week follow-up tests revealed that the memory of extinction was intact in these mice (with prefrontal LTP) and in control mice displaying prefrontal LTP-like changes, whereas control mice that did not exhibit such changes displayed a return of freezing to the CS. The results suggest that after extinction the lack of depression-LTP-like conversion sequence in the MPFC synaptic efficacy may profoundly alter the process of consolidation.

Key words: fear conditioning; extinction; long-term depression; long-term potentiation; medial prefrontal cortex; mouse

ing of the mPFC, are not maintained in up to $40 \%$ of individuals treated for PTSD (Tarrier et al., 1999). Furthermore, a conversion from depression to potentiation of prefrontal neuronal activity develops in some patients after therapeutic extinction of PTSD symptoms (Fernandez et al., 2001). This leads to the hypothesis that the absence of such prefrontal changes after a complete extinction of PTSD symptoms is associated with the return of those symptoms. Third, data from animal studies suggest that although the mPFC is not necessary for extinction learning per se (Gewirtz et al., 1997; Quirk et al., 2000; Vouimba et al., 2000), it may be required for the development of long-term memory of extinction (Quirk et al., 2000). Fourth, we have recently observed in mice that extinction of learned fear is associated with a sequence of depression and potentiation of synaptic efficacy in the mPFC (Herry et al., 1999).

From these observations, we hypothesized that long-term maintenance (consolidation) of the treatment effects (extinction learning) may, at least in part, depend on the conversion from depression to long-lasting normalization or potentiation of prefrontal synaptic efficacy. Hence, the present study was undertaken to evaluate the possibility that long-term depression (LTD) in the $\mathrm{mPFC}$ would favor a return of conditioned fear, whereas prefrontal long-term potentiation (LTP) would characterize long-term maintenance of extinction of learned fear. With this objective, LTD and LTP in the mPFC were induced by low-frequency stimulation (LFS) and high-frequency stimulation (HFS) of the mediodorsal thalamic nucleus (MD), respectively. Mice were conditioned to express freezing behavior to a tone conditioned stimulus (CS) previously paired with footshock unconditioned 
stimulus (US). The reduction or complete extinction of this response was induced by repeated presentations of the CS without the US. Treatments for inducing long-term changes in synaptic plasticity in the mPFC were administered (LFS or HFS of the MD) before extinction sessions.

\section{MATERIALS AND METHODS}

Subjects and surgery

Subjects were 4-month-old male C57BL/6 mice (IFFA Credo, Lyon, France). They were individually housed in Plexiglas cages and were maintained on a free feeding regimen with a $12 \mathrm{hr}$ light/dark schedule. All studies took place during the light portion of the cycle. The experiments were performed in accordance with the European Communities Council Directive (86/609/EEC).

Using avertin (made up as $1.25 \mathrm{ml}$ avertin concentrate, i.e., $100 \mathrm{gm}$ of tribromoethanol dissolved in $62 \mathrm{ml}$ of tertiary amyl alcohol, added to $5 \mathrm{ml}$ of absolute alcohol and $62.5 \mathrm{ml}$ of physiological saline; $10 \mathrm{ml} / \mathrm{kg}$, i.p.) as anesthetic and conventional surgery techniques, mice were ipsilaterally implanted with electrodes made of twisted platinum-iridium wires $(90$ $\mu \mathrm{m}$ diameter) insulated except at the tip. To reduce cell damage, the tips of the wires were sectioned at an angle. Electrodes were positioned in the MD (stimulation: $0.8 \mathrm{~mm}$ posterior to bregma and $0.3 \mathrm{~mm}$ lateral to midline) and the mPFC (recording: $2.4 \mathrm{~mm}$ anterior to bregma, $0.4 \mathrm{~mm}$ lateral to midline) at a location generating a maximal amplitude of the prefrontal field potential (Herry et al., 1999). The entire miniature system was fixed in place onto the skull with dental cement. Subjects were then allowed to recover in their home cages in the colony room for at least $7 \mathrm{~d}$.

\section{Electrophysiological recording procedures}

Electrophysiological recordings were performed in a square transparent Plexiglas box $(18 \mathrm{~cm}$ side $\times 23 \mathrm{~cm}$ high) with a gray plastic floor. The Plexiglas box (recording context) was placed inside a sound-attenuating and temperature-regulated Plexiglas cubicle located in a room that was separated from the main room (where the experiment was guided via computers). The recording context was washed with $1 \%$ acetic acid before and after each session. Electrophysiological activity was recorded through junction field effect transistor (JFET) operational amplifiers connected to the headstage to minimize artifacts caused by head movement. Cables from the JFET were relayed at the top of the recording chamber by a multichannel rotating connector. This system allowed the animals free movement within the recording chamber. Prefrontal field potentials evoked by single-pulse thalamic stimulation $(0.1 \mathrm{msec}$ rectangular biphasic pulses) were sent to an amplifier (gain, 1000×; bandpass $1-10 \mathrm{kHz}$ ) and recorded (pClamp6 software; Texas Instruments) for off-line analysis. Stimulus intensity was chosen (from the baseline inputoutput curves: $60-600 \mu \mathrm{A}$ ) as that which produced a response amplitude $\sim 60-70 \%$ of the maximal level.

\section{Behavioral recording procedures}

The behavior of each mouse was continuously monitored and videotaped to score freezing behavior. During CS presentation, a mouse was considered to freeze when it adopted a motionless posture, refraining from all but respiratory movements (Blanchard and Blanchard, 1969). Freezing was scored using a time-sampling procedure. Specifically, every $2 \mathrm{sec}$ (before the first CS presentation: $120 \mathrm{sec}$ observation; during each CS presentation: $20 \mathrm{sec}$ observation), the mouse was determined to be freezing or not freezing by an experimenter who was blind to the experimental history of each mouse.

\section{Conditioning apparatus}

Training took place in a conditioning context consisting of a gray plastic cylinder $(15.5 \mathrm{~cm}$ diameter $\times 14 \mathrm{~cm}$ high) with a shock grid floor made of stainless steel rods. The conditioning context was placed inside the recording context to preserve, from conditioning to extinction sessions, features of the tone $(2.5 \mathrm{kHz}, 80 \mathrm{~dB})$ generated by a speaker at the top of the recording context. The shock grid was connected to a current generator and scrambler to provide a $1 \mathrm{sec}, 0.9 \mathrm{~mA}$ footshock. The conditioning box and the floor were cleaned with $70 \%$ ethanol before and after each session.

\section{Procedure}

Experiment 1. After recovery from surgery, mice were habituated to being transported (from the animal house to the experimental room) and to connection and disconnection of the miniature headstage over a $4 \mathrm{~d}$ period. After habituation, baseline electrophysiological responses were established over a 2 d period (one recording session per day; each recording corresponding to an average of seven field potentials recorded at $0.2 \mathrm{~Hz}$; days 1 and 2). Six days later (day 8), a third recording session was made to test the stability of responses before auditory fear conditioning. Mice were then divided into two groups. Each mouse was placed into the conditioning chamber, but mice of only one group $(n=13)$ were conditioned to acquire fear in response to a $20 \mathrm{sec}$ tone CS that was paired with footshock US (four CS-US pairings; intertrial interval: $60-180 \mathrm{sec}$ ). The onset of the US coincided with the offset of the CS. The other group (control; $n=5$ ), which served as a control for the stability of electrophysiological recordings over the whole experiment period, received an equivalent number of tone presentations (intertrial interval: $60-180 \mathrm{sec}$ ), but without the US. From the next day (day 9), each mouse of the two groups was placed back into the recording chamber, and the CS was presented alone starting $10 \mathrm{~min}$ after entry (four CSs per session; intertrial interval: $60-180 \mathrm{sec}$ ) to induce extinction of learned fear (three sessions: days 9-11). To examine the effect of prefrontal LTD induction on the evolution of extinction of freezing behavior between days, the conditioned group was divided into two subgroups. One subgroup received LFS (a train of 1200 pulses at $2 \mathrm{~Hz}$ ) to the MD, a protocol known to induce LTD in the dorsal mPFC (Herry et al., 1999), 10 min before each of the subsequent sessions of extinction (sessions 2-3; LFS group; $n=6$ ). The other group did not receive LFS (NLFS group; $n=7$ ). Field potentials in $\mathrm{mPFC}$, evoked by MD stimulation, were recorded (seven responses recorded at $0.2 \mathrm{~Hz}$ ) before the first session of extinction (after the first $9 \mathrm{~min}$ in the recording chamber: recording D9a), during each CS-alone presentation (first session: recording D9b; second and third sessions: recordings D10 and D11, respectively) and $24 \mathrm{hr}$ (day 12) after the third session of extinction (recording D12). The extinction procedure used in this experiment was chosen to prevent development of prefrontal LTP-like changes between days.

Experiment 2. It was designed to test the effects of MD thalamic HFS (10 trains, $10 \mathrm{sec}$ apart, of 50 pulses at $250 \mathrm{~Hz}$ ) on prefrontal synaptic excitability and consequently on extinction of learned fear. For this purpose, after the habituation period, mice were divided into two groups. In the first group (HFS-test; $n=7$ ), four baseline recordings were performed at $16,8,4$, and 2 min before the HFS. Post-HFS recordings were performed $2,4,8,16$, and 32 min later (seven field potentials at 0.2 $\mathrm{Hz}$ per recording). In the second group $(n=17)$, electrophysiological baseline and conditioning were conducted as for experiment 1 . However, $24 \mathrm{hr}$ after conditioning (day 9), animals were subdivided into two groups, one group received HFS 32 min before the extinction session (HFS group; $n=8$ ). The other group served as control (NHFS group; $n=9$ ). The extinction procedure comprised a single session (16 CSalone presentations; intertrial interval: $60-180 \mathrm{sec})$. Long-term maintenance of extinction was tested 1 week later (day 16) using a single session of four CS-alone presentations (intertrial interval: $60-180 \mathrm{sec}$ ). The extinction procedure of the second experiment was chosen for two main reasons. First, to examine whether pre-extinction mPFC LTP would inhibit expression of fear as we previously observed in our laboratory for the lateral septum (Vouimba et al., 1998, 1999). This question was raised because lesions of each of these structures potentiate freezing behavior (mPFC: Morgan and LeDoux, 1995; Vouimba et al., 2000; lateral septum: Vouimba et al., 1998). Consequently, we wished to test whether inhibition of fear expression would facilitate extinction within a single session. Second, we hypothesized that a single session of extinction with 16 trials would induce complete extinction of freezing but would produce only weak changes in synaptic plasticity in the $\mathrm{MPFC}$, because in our previous study 25 trials were necessary to induce from day 1 to day 2 conversion from depression to potentiation (Herry et al., 1999). In this way, we examined whether mice with $\mathrm{mPFC}$ LTP would display a better maintenance of extinction as compared with mice without such changes in the mPFC.

\section{Histology and data analysis}

On completion of the experiments, mice were deeply anesthetized, and the electrode tip placements were marked by passing $0.5 \mathrm{~mA}$ current for $20 \mathrm{sec}$. The placement of the electrodes was then verified by standard histological methods. All electrophysiological and behavioral data were expressed as means and SEM and ANOVA followed by post hoc Scheffé $F$ tests. 

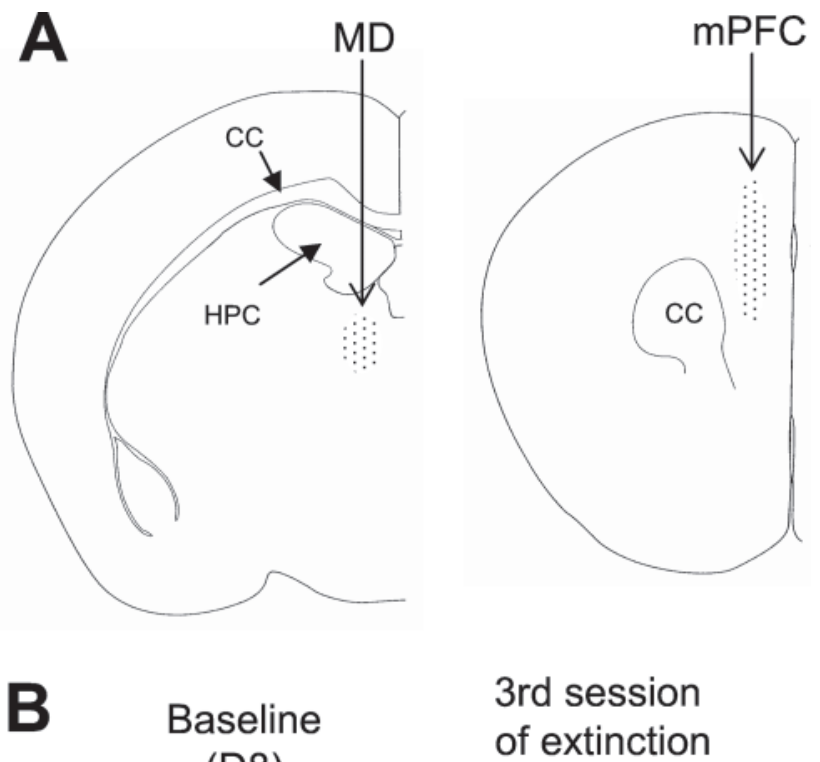

(D8)

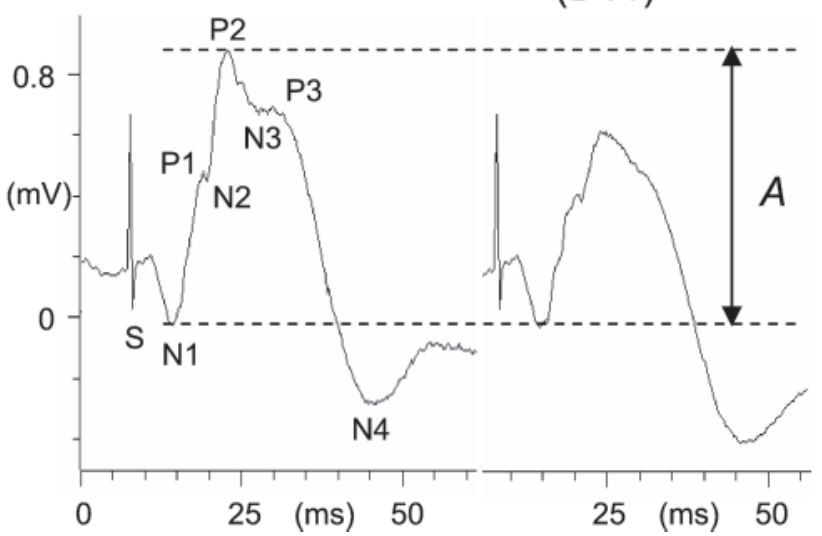

Figure 1. A, Diagrams of coronal sections of the mouse brain showing electrode placements (dotted areas) in the MD (left) and the MPFC (right). $C C$, Corpus callosum; $H P C$, dorsal hippocampus. $B$, Example of changes in field potential amplitude in a mouse from the LFS group. These representative responses were recorded during the establishment of the baseline (left) and the third sessions of extinction (right). Changes in prefrontal excitability corresponded to changes in the amplitude of the N1-P2 complex (the amplitude $A$, between the two dotted lines, represents the reference amplitude of the N1-N2 complex).

\section{RESULTS}

\section{Histology}

The locations of the recording sites in the MD and the $\mathrm{mPFC}$ are shown in Figure $1 A$. The histological analysis revealed that all mice had correct electrode placements with the recording electrode mainly in the prelimbic area of the mPFC.

\section{Experiment 1: effects of thalamic LFS}

\section{Electrophysiology}

As previously described (Herry et al., 1999), MD stimulation evoked a field response in the mPFC characterized (Fig. $1 B$ ) by two initial complexes (N1-P1: 5-10 msec; N2-P2: 10-16 msec) followed by two other complexes (P2-N3: 15-21 msec; P3-N4: 22-36 msec). Plasticity of prefrontal synaptic excitability was assessed by changes in the N1-P2 (including both N1-P1 and N2-P2 complexes) amplitude (Fig. $1 B$ ), which represent changes in the probability of discharging target cells (Pirot et al., 1994;

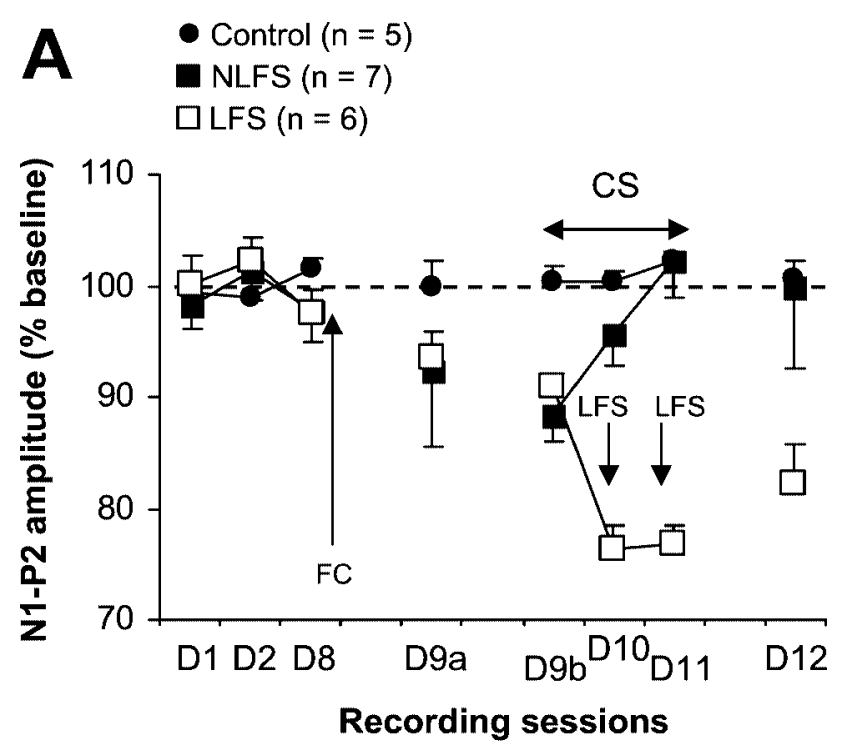

B

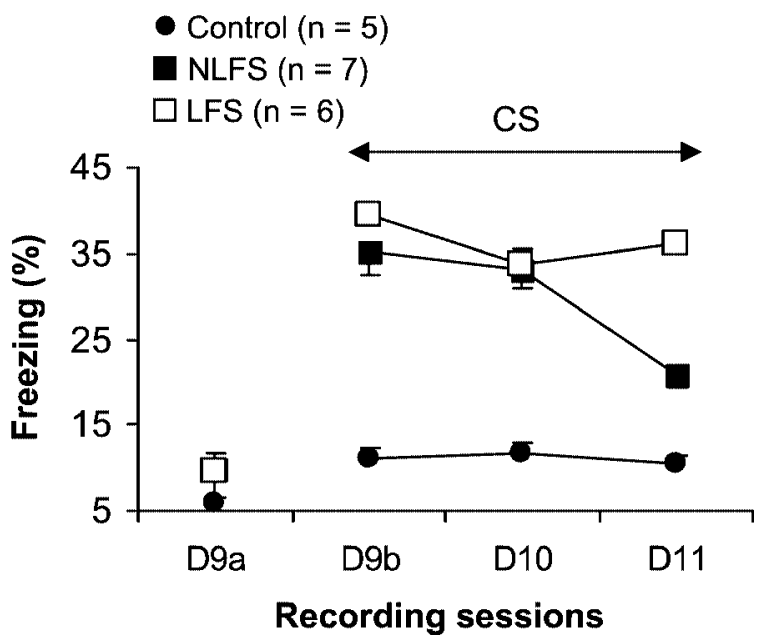

Figure 2. A, Mean percentage of changes in N1-P2 amplitude ( \pm SEM) during the $3 \mathrm{~d}$ of baseline recording $(D 1-D 8)$, before the first session (D9a), during sessions $(D 9 b-D 11)$, and $24 \mathrm{hr}$ after the last session (D12) of extinction in conditioned mice that received (LFS group) or did not receive (NLFS group) thalamic LFS and nonconditioned mice (control group). Fear conditioning $(F C)$ took place after the last baseline recording $(D 8) . B$, Mean percentage of freezing behavior ( \pm SEM) in the three groups during the $120 \mathrm{sec}$ period preceding the first CS-alone presentation $(D 9 a)$ and sessions of CS-alone presentations $(D 9 b-D 11)$.

Herry et al., 1999). The amplitude of the N1-P2 component was stable across the $3 \mathrm{~d}$ of baseline recording sessions (Fig. 2A) $\left(\mathrm{D} 1-\mathrm{D} 8 ; F_{(2,34)}=0.63\right)$. Twenty-four hours after the conditioning session, when placed back into the recording chamber (Fig. $2 \mathrm{~A}$, $D 9 a)$, mice of the conditioned groups displayed a slight but nonsignificant decrease in the N1-P2 amplitude $\left(F_{(3,36)}=2.21\right.$; NS). Presentation of the tone (Fig. $2 A, D 9 b$ ) resulted in an additional and significant decrease in the N1-P2 amplitude with respect to both baseline values (NLFS group: $F_{(3,18)}=3.69, p<$ 0.05; LFS group: $\left.F_{(3,15)}=4.05, p<0.05\right)$ and values of the control group (NLFS group: $F_{(1,10)}=8.76, p<0.05$; LFS group: $F_{(1,9)}=$ $12.06, p<0.05)$. This effect was followed by a progressive recovery toward normal levels of synaptic excitability across the two subsequent sessions of extinction in mice that did not receive LFS. The fact that the decrease in the N1-P2 amplitude was not 
converted into long-lasting potentiation, as shown previously (Herry et al., 1999), was probably related to the particular schedule that we used here for extinction. In our previous experiments extinction was induced using a massed-trials schedule (25 trials per day), whereas here extinction was induced using a spaced schedule (four trials per day). However, both schedules were associated with the suppression of depression of mPFC synaptic efficacy, whereas the depression was maintained in mice that received LFS before the second and third sessions of CS-alone presentations. These animals (i.e., LFS group) displayed even a larger decrease in the N1-P2 amplitude as compared with the previous depression $\left(F_{(2,10)}=10.78 ; p<0.01\right)$. The day after (Fig. 2A, D12), the N1-P2 amplitude remained at baseline levels in mice in the NLFS group (these values were similar to those obtained for the Control group), whereas the decrease in N1-P2 amplitude in mice that received LFS was still present and significant as compared with both the NLFS group $\left(F_{(1,11)}=10.73 ; p<\right.$ $0.01)$ and controls $\left(F_{(1,9)}=43.44 ; p<0.001\right)$.

Results from the $L F S$ group also reveal that during the extinction procedure (with this spaced schedule), mPFC synapses are "inclined" to exhibit LTD after thalamic LFS, whereas in a normal situation their can either display LTD or potentiation after the same type of stimulation (Herry et al., 1999).

\section{Behavior}

The percentage of freezing behavior was measured during the $120 \mathrm{sec}$ period preceding the first CS-alone presentation and during the $20 \mathrm{sec}$ period of each CS-alone presentation. Mice of the control group exhibited stable low levels of freezing over all recording sessions $(F<1)$. Both LFS and NLFS groups displayed also low levels of freezing before the extinction procedure (Fig. 2B), indicating the absence of contextual generalization of fear (extinction took place in a context that was different from the conditioning chamber). Freezing in the three groups did not differ during the $120 \mathrm{sec}$ period preceding the first CS-alone presentation $(F<1)$. However, mice of the two conditioned groups (LFS and NLFS) displayed a high percentage of freezing during the first two sessions of CS-alone presentations (Fig. 2B). The next day, mice of the NLFS group exhibited less freezing than did the LFS group. A two-factor repeated measures ANOVA performed on these data (three levels: D9b-D11) showed a significant effect of conditions $\left(F_{(2,15)}=40.01 ; p<0.001\right)$, with an effect of sessions $\left(F_{(2,30)}=16.95 ; p<0.001\right)$. The interaction between condition and session was also significant $\left(F_{(4,30)}=9.92 ; p<0.001\right)$. A direct between-groups comparison showed that both NLFS and LFS groups displayed significantly higher percentages of freezing during each session of CS-alone presentations as compared with control mice (all $p<0.001$ ). The two conditioned groups differed from each other only on the last session $\left(F_{(1,11)}=15.48 ; p<\right.$ $0.001)$. A one-factor ANOVA with repeated measures performed on data from the NLFS group indicated a significant reduction of freezing over sessions of CS-alone presentations $\left(F_{(2,12)}=39.75\right.$; $p<0.001)$. In contrast, mice subjected to LFS of the MD presented strong resistance to extinction of learned freezing (Fig. $2 B$ ) (this is also indicated by a nonsignificant effect of sessions).

\section{Experiment 2: effect of thalamic HFS} Electrophysiology

Figure 3 illustrates the effects of HFS on the evoked response in the mPFC. All mice in the HFS-test group displayed stable baseline response $(F=1)$. At 2 min after HFS, an initial decrease was observed, whereas subsequent recordings revealed a return to
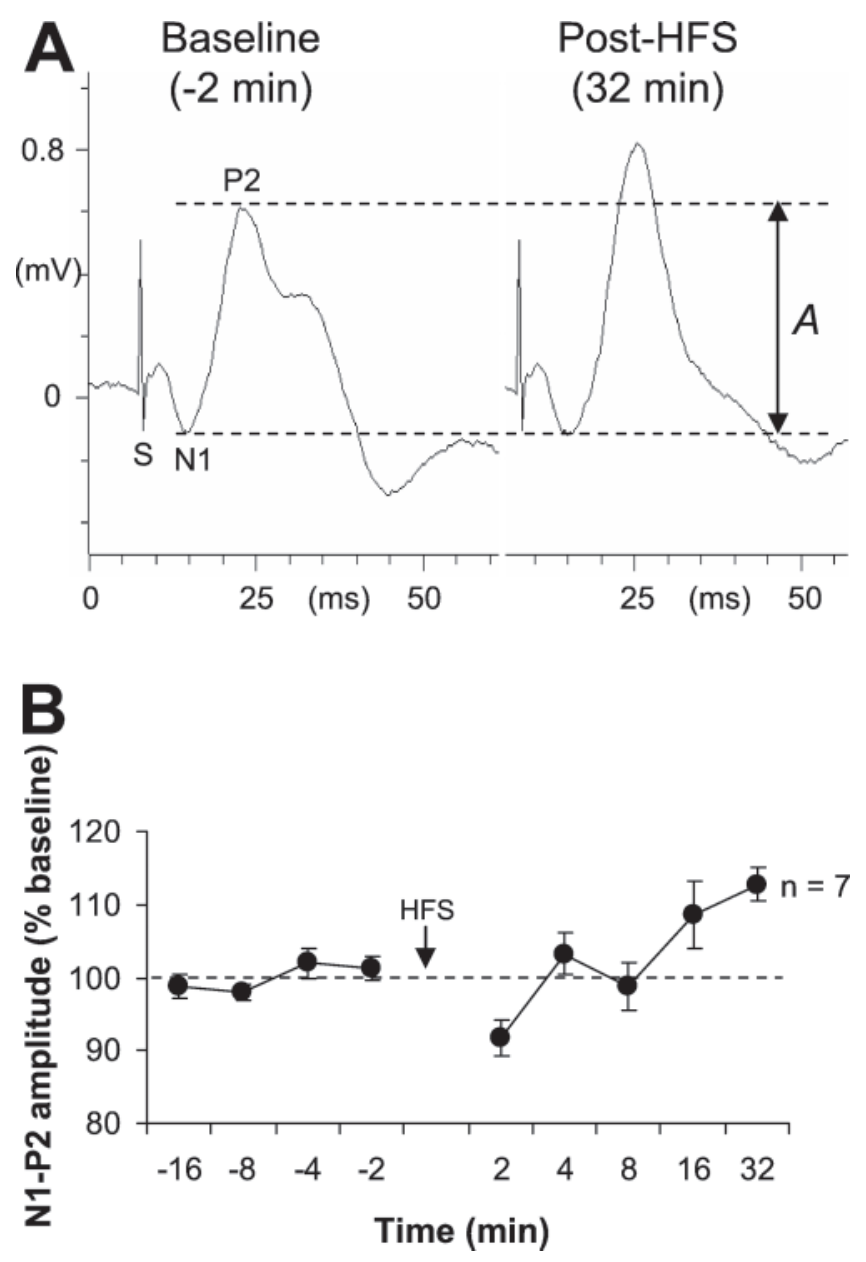

Figure 3. A, Example of change in MD-mPFC-evoked response after thalamic HFS. These representative responses were recorded during the establishment of the baseline and 32 min after thalamic HFS (the amplitude between the two dotted lines represents the reference amplitude of the N1-P2 complex). B, Mean percentage changes in N1-P2 amplitude ( \pm SEM) during different recording sessions (before and after thalamic HFS).

baseline levels, which was then followed by potentiation. One-way ANOVA (nine levels) indicated an effect of $\operatorname{HFS}\left(F_{(8,48)}=6.15\right.$; $p=0.0001)$. Post hoc Scheffé $F$ tests indicated that the depression ( $2 \mathrm{~min}$ ) reached statistical significance as compared with baseline levels $\left(F_{(4,24)}=4.29 ; p<0.01\right)$. Potentiation was significant only at the 32 min post-HFS delay $\left(F_{(4,24)}=9.12 ; p=0.0001\right)$. This delay was, therefore, chosen for testing the effects of thalamic HFS on both the rate of extinction of learned freezing and maintenance of extinction.

Figure $4 A$ shows changes in the MD-mPFC-evoked responses during the extinction of freezing to the tone CS in mice that had received previous thalamic HFS (HFS group) and control mice (NHFS group). Recordings made to establish baseline values were stable in the two groups (HFS and NHFS). Thalamic HFS induced an increase in amplitude, but this was not significant compared with both baseline values and values obtained with NHFS mice (D9a). Significant changes (potentiation) were observed for the final two blocks of CS-alone presentations (D9d: $F_{(3,21)}=20.46 ; p=0.0001$; D9e: $\left.F_{(3,21)}=7.75 ; p=0.001\right)$. Some potentiation appeared to be present 1 week later, but this was significantly higher than baseline values only during the CS test 


$$
\begin{array}{cl}
\mathbf{O N H F S} 1(n=4) \\
\\
\quad \operatorname{NHFS} 2(n=5) \\
\square \operatorname{HFS}(n=8)
\end{array}
$$

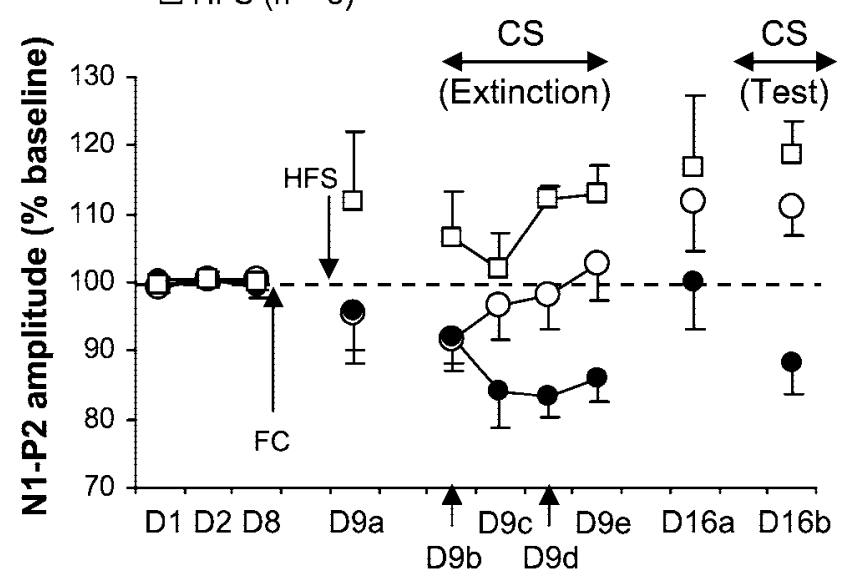

\section{Recording sessions}
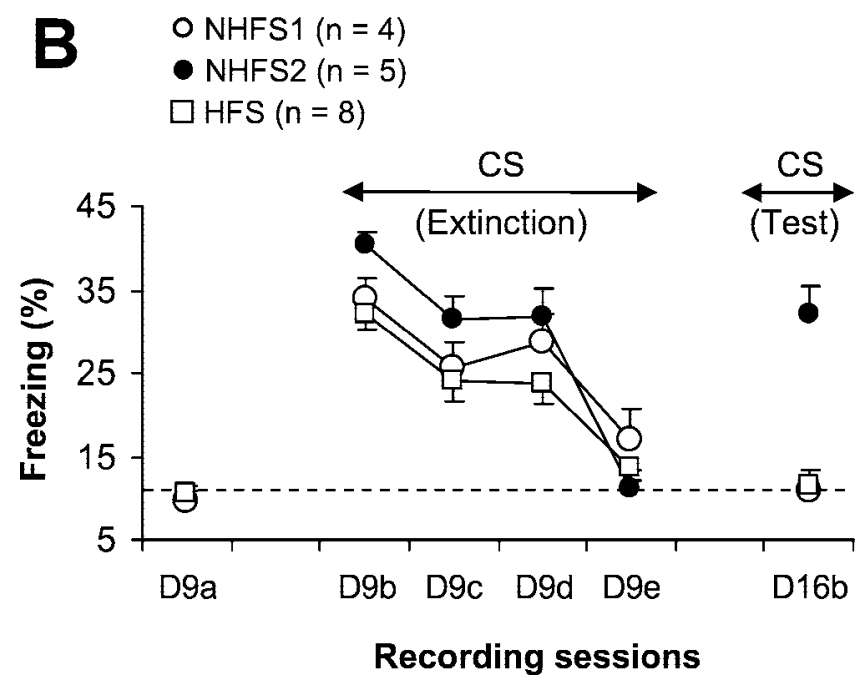

Figure 4. A, Mean percentage of changes in N1-P2 amplitude ( \pm SEM) during the $3 \mathrm{~d}$ of baseline recording (D1-D8), before the first session $(D 9 a)$ and during sessions $(D 9 b-D 9 e)$ of extinction, before $(D 16 a)$ and during $(D 16 b)$ the follow-up test in conditioned mice that received HFS before extinction (HFS group) and their controls that did not receive HFS (NHFS1 and NHFS2 groups). Fear conditioning $(F C)$ took place after the last baseline recording (D8). B, Mean percentage of freezing behavior $( \pm$ SEM) in the three groups during the $120 \mathrm{sec}$ period preceding the first CS-alone presentation $(D 9 a)$, during CS-alone presentations $(D 9 b-D 9 e)$, and during the 1 week follow-up test of CS-alone presentations $(D 16 b)$. The dotted line represents mean level of freezing displayed by nonconditioned mice (experiment 1 ).

$\left(F_{(3,21)}=11.99 ; p=0.0001\right)$. In the NHFS group, a slight and nonsignificant decrease in the N1-P2 amplitude was observed before the extinction session. CS-alone presentations (first block of four trials) (Fig. 4A, D9b) produced an additional and significant decrease $\left(F_{(3,24)}=5.78 ; p<0.01\right)$. However, examination of individual changes in field potentials in these mice revealed that during the subsequent blocks of CS-alone presentations, only five subjects (NHFS2) of the nine exhibited a persistent depression in the N1-P2 amplitude (all $p<0.01$ ). Recordings made 1 week later showed that the amplitude of the N1-P2 component in these mice returned to baseline levels, but when the CS was presented again, a significant decrease was once again observed $\left(F_{(3,12)}=\right.$ 5.18; $p<0.05)$. The remaining subjects (NHFS1) showed rapid recovery of normal excitability (as compared with the first block of CS-alone presentations). Recordings made 1 week later revealed the existence of potentiation that became significant during the CS-alone presentations $\left(F_{(3,9)}=5.11 ; p<0.03\right)$. A direct between-groups comparison indicated that the HFS group differed from the nonpotentiated group (NHFS2) on the two last sessions of extinction and on the 1 week follow-up session of CS-alone presentations (all $p<0.02$ ). They differed from the NHFS1 group only on the session D9d (Fig. $4 A$ ) of extinction $(p<0.01)$. The two NHFS groups differed from each other on the two last sessions of extinction and on the follow-up session (all $p<0.03)$.

\section{Behavior}

The three groups displayed low levels of freezing (similar to those obtained for the nonconditioned mice; experiment 1 ), and did not differ from each other $(F<1)$ during the $120 \mathrm{sec}$ period preceding the first CS-alone presentation. These behavioral data indicate also, as for experiment 1, the absence of contextual generalization of fear. However, high levels of freezing were observed in all groups during the CS-alone presentations (Fig. 4B). Extinction of freezing behavior to the tone CS occurred at similar rates in all mice. During the last four CS-alone presentations, all mice exhibited levels of freezing that were equivalent to those displayed by nonconditioned mice (experiment 1 ), indicating that our extinction procedure produced a complete extinction of freezing to the tone CS. These data (with electrically potentiation), as well as those obtained previously (behaviorally induced potentiation; Herry et al., 1999), show that potentiation of mPFC synaptic excitability has no effect on the rate of extinction of freezing behavior toward a tone CS. However, 1 week later, CS-alone presentations reactivated learned freezing response to the tone CS in NHFS2 mice (i.e., mice that also displayed depression in the amplitude of the thalamoprefrontal responses), whereas freezing remained at basal levels in both NHFS1 (natural potentiation) and HFS mice. A two-factor repeated measures ANOVA (three groups $\times$ four sessions: D9b-D9e) revealed a highly significant main effect of sessions $\left(F_{(3,42)}=37.04 ; p=\right.$ $0.0001)$. The main effect of groups and the interaction between group and session were not significant. However, a main effect of groups was observed with ANOVA performed on data from the 1 week follow-up test $\left(F_{(2,14)}=35.42 ; p=0.0001\right)$. Mice in the NHFS2 group differed from mice in both NHFS1 $\left(F_{(1,7)}=24.78\right.$; $p=0.001)$ and HFS group $\left(F_{(1,11)}=66.57 ; p=0.0001\right)$, whereas the NHFS1 and HFS groups did not differ from each other $(F<$ 1 ). When the follow-up data were compared with values obtained from the last block of extinction, a statistically significant difference was found only for the NHFS2 mice $\left(F_{(1,4)}=26.96 ; p<\right.$ $0.01)$.

\section{DISCUSSION}

The major findings of the present study are as follows: (1) the persistence of synaptic depression (LTD) in the mPFC during the extinction of learned fear is associated with the return of learned fear (emotional perseveration), whereas (2) LTP in the mPFC is associated with the maintenance of extinction. These findings suggest that long-lasting increase of synaptic efficacy in the mPFC may be involved in mechanisms leading to long-term maintenance 
of extinction probably by inhibiting emotional perseveration after an extinction procedure.

\section{Emotional perseveration}

Neurons within the mPFC have been implicated in the inhibition of inappropriate behaviors (Fuster, 1989; Hauser, 1999). One related question that has emerged concerns whether the mPFC is also implicated in the inhibition of emotional perseveration, such as inhibition of perseverative freezing to a tone CS that is repetitively presented without footshock US (Morgan et al., 1993; Morgan and LeDoux, 1995). Accordingly, dysfunction in prefrontal inhibitory mechanisms may lead to a resistance to extinction of fear. However, recent experiments, using lesion methodologies, have not readily provided evidence in support of this hypothesis (Gewirtz et al., 1997; Vouimba et al., 2000).

Previous studies, using electrophysiological approaches, have shown that a tone CS previously paired with footshock produces depressed excitability in the mPFC (Garcia et al., 1999; Herry et al., 1999). The present data suggest that the persistence of this prefrontal excitability depression may be involved in resistance to extinction (or emotional perseveration). First, we observed that excitability in the MD-mPFC pathway returned to baseline levels during the extinction of conditioned freezing in mice that did not receive LFS of the MD. This indicates that extinction was associated with a change in direction of plasticity in MD-mPFC transmission. Second, thalamic LFS applied before the second and third session of extinction (1) blocked a return to the baseline levels of excitability in the MD-mPFC circuits, and (2) produced a complete return of fear during the third session of extinction. If thalamic LFS has a specific effect on freezing expression (e.g., a potentiation of freezing behavior), this effect would have been observed after the first application of LFS. However, during the second session of extinction, the levels of freezing in mice that received thalamic LFS did not differ from mice that did not receive such stimulation. It seems more likely that thalamic LFS promoted the return of freezing at its maximal acquired levels (35-40\%) instead of potentiating freezing behavior to the CS. At present it is difficult to establish a causal role of prefrontal LTD in the return of freezing at maximal previously acquired levels. Indeed, thalamic LFS could have induced changes in synaptic plasticity in other brain structures in addition to the mPFC. The effects of thalamic LFS could, therefore, be attributable to either these changes outside the mPFC, changes in the mPFC, or both. However, evidence for association between changes in prefrontal neuronal activity and emotional perseveration is also provided by our second experiment in which we observed that mice that presented natural maintenance of depression in prefrontal synaptic transmission during the extinction procedure also displayed a return of fear 1 week later.

Data of the second experiment also show that depression of synaptic excitability in the mPFC does not interfere with extinction within a session but rather between days. This is consistent with a recent study showing that lesions of the mPFC do not block extinction of freezing behavior within a session but promote a return of freezing the next day (Quirk et al., 2000).

\section{Long-term maintenance of extinction}

The extinction of a learned response is considered as new learning, mainly because it is thought to require the formation of a new memory (i.e., the CS is no longer followed by the US; see Falls et al., 1992, for more details). Extinction learning can be longlasting (Tarrier et al., 1999), indicating that information relative to extinction can be stored in long-term memory via a process referred to as consolidation. Lesion studies suggest that the mPFC may be involved in the subsequent consolidation but not in the initial learning of extinction (Quirk et al., 2000). From our electrophysiological studies (see also Herry et al., 1999), we suggest that the depression-potentiation conversion sequence in prefrontal synaptic plasticity, which occurs between days and not within a session, might be a crucial neurophysiological mechanism underlying the consolidation function of the mPFC rather than the extinction learning per se. First, all mice in experiment 2 displayed an extinction of freezing to the CS independently of the evolution of synaptic plasticity in the mPFC (maintenance of depression, tendency to normalization or potentiation) within the extinction session. Second, only mice that developed changes in the direction of synaptic plasticity during extinction also displayed maintenance of extinction. Third, none of the mice that received thalamic HFS showed a return of fear 1 week after extinction. This suggests that LTP-like changes in the mPFC synaptic plasticity may either be directly involved in the long-term maintenance of extinction or represent a prerequisite mechanism for consolidation. Even if recent findings support the involvement of the mPFC in the consolidation of extinction (Quirk et al., 2000), the precise function of the mPFC (facilitation or physical support of consolidation) remains unclear. Conceptually, it is assumed that the formation of long-term memory involves modulation of gene expression (Davis and Squire, 1984). For further studies, it will be of particular relevance to our understanding to investigate whether injection of inhibitors of protein synthesis within the mPFC during the learning phase of extinction and/or immediately afterward blocks the formation of long-term but not the short-term memory of extinction.

\section{Clinical implications}

Studies using positron emission tomography, superimposed on a magnetic resonance reference image, have shown that reexposure to traumatic material is accompanied by a decrease in blood flow in the mPFC of PTSD patients (Bremner et al., 1999a,b; Fernandez et al., 2001). On the one hand, we suggest that severe stress can precipitate LTD-like changes in the MPFC that may in turn precipitate long-lasting changes in affect in vulnerable individuals (Yehuda et al., 1998; McFarlane, 2000). Consequently, syndromes such as PTSD, which can in some patients be resistant to treatment (Friedman, 1997; Tarrier et al., 1999), may persevere (recovery of extinguished fear) because of the perseveration of plasticity acquired by exposure of individuals to traumatic stress. On the other hand, significant increases in blood flow as compared with basal levels have been observed in the mPFC after therapeutic extinction of PTSD symptoms (Fernandez et al., 2001). These data are in accordance with our observations in mice regarding the development of prefrontal depression-potentiation conversion sequence during extinction. Together with the present findings, this suggests that trauma-related plasticity in certain brain circuits, including the $\mathrm{mPFC}$, should change in direction to allow maintenance of improvement after exposure or cognitive therapy (Tarrier et al., 1999). Finally, the relationship between the prefrontal depression-potentiation sequence and the maintenance of extinction needs to be confirmed in humans by follow-up studies.

In summary, conditioned fear induces depression of prefrontal synaptic excitability. During extinction of the learned fear, there is either a potentiation (which develops between days after a massed-trials schedule) or a normalization (which can develop 
within a session) or a maintenance of depression of prefrontal synaptic excitability. However, only potentiation of excitability, even induced artificially by HFS of the MD (MD inputs to the mPFC are bilaterally driven; Kuroda et al., 1998), is associated with the maintenance of extinction.

\section{REFERENCES}

Blanchard RJ, Blanchard C (1969) Crouching as an index of fear. J Comp Physiol Psychol 67:370-375.

Bremner JD, Staib LH, Kaloupek D, Southwick SM, Sooufer R, Charney DS (1999a) Neural correlates of exposure to traumatic pictures and sound in Vietnam combat veterans with and without posttraumatic stress disorder: a positron emission tomography study. Biol Psychiatry 45:806-816.

Bremner JD, Narayan M, Staib LH, Southwick SM, McGlashan T, Charney DS (1999b) Neural correlates of memories of childhood sexual abuse in women with and without posttraumatic stress disorder. Am J Psychiatry 156:1787-1795.

Davis HP, Squire LR (1984) Protein synthesis and memory: a review. Psychol Bull 96:518-559.

Ettedgui E, Bridges M (1985) Posttraumatic stress disorder. Psychiatr Clin North Am 8:89-103.

Falls WA, Miserendino MJ, Davis M (1992) Extinction of fearpotentiated startle: blockade by infusion of an NMDA antagonist into the amygdala. J Neurosci 12:854-863.

Fernandez M, Pissiota A, Frans O, Knorring L V, Fischer H, Fredrikson M (2001) Brain function in patient with torture related post-traumatic stress disorder before and after fluoxetine treatment: a positron emission tomography provocation study. Neurosci Lett 297:101-104.

Friedman MJ (1997) Posttraumatic stress disorder. J Clin Psychiatry 58[Suppl 9]:33-36.

Fuster JM (1989) The prefrontal cortex, anatomy, physiology and neuropsychology of the frontal lobe. New York: Raven.

Garcia R (2001) Stress, hippocampal plasticity, and spatial learning. Synapse 40:180-183.

Garcia R, Vouimba RM, Baudry M, Thompson RF (1999) Changes in medial prefrontal cortex activity during the expression of conditioned fear are mediated by amygdala. Nature 402:294-296.

Gewirtz JC, Falls WA, Davis M (1997) Normal conditioned inhibition and extinction of freezing and fear-potentiated startle following electrolytic lesions of medial prefrontal cortex in rats. Behav Neurosci 111:712-726.

Hauser MD (1999) Perseveration, inhibition and the prefrontal cortex: a new look. Curr Opin Neurobiol 9:214-222.

Herry C, Vouimba RM, Garcia R (1999) Plasticity in the thalamo- prefrontal cortical transmission in behaving mice. J Neurophysiol 82:2827-2832.

Kuroda M, Yokof ujita J, Murakami K (1998) An ultrastructural study of the neural circuit between the prefrontal cortex and the mediodorsal nucleus of the thalamus. Prog Neurosci 54:417-458.

Markowitsch HJ, Kessler J, Van Der Ven C, Weber-Luxenburger G, Albers M, Heiss WD (1998) Psychic trauma causing grossly reduced brain metabolism and cognitive deterioration. Neuropsychologia 36:77-82.

McFarlane AC (2000) Posttraumatic stress disorder: a model of the longitudinal course and the role of risk factors. J Clin Psychiatry 61[Suppl 5]:15-20.

Moradi AR, Doost HT, Taghavi MR, Yule W, Dalgleish T (1999) Everyday memory deficits in children and adolescents with PTSD: performance on the Rivermead Behavioural Memory Test. J Child Psychol Psychiatry 40:357-361.

Morgan MA, LeDoux JE (1995) Differential contribution of dorsal and ventral medial prefrontal cortex to acquisition and extinction of conditioned fear in rats. Behav Neurosci 109:681-688

Morgan MA, Romanski LM, LeDoux JE (1993) Extinction of emotional learning: contribution of medial prefrontal cortex. Neurosci Lett 163:109-113.

Pirot S, Jay TM, Glowinski J, Thierry AM (1994) Anatomical and electrophysiological evidence for excitatory amino acid pathway from the thalamic mediodorsal nucleus to the prefrontal cortex in the rat. Eur J Neurosci 6:1225-1234.

Quirk GJ, Russo GK, Barron JL, Lebron K (2000) The role of ventromedial prefrontal cortex in the recovery of extinguished fear. J Neurosci 20:6225-6231.

Tarrier N, Sommerfield C, Pilgrim H, Humphreys L (1999) Cognitive therapy or imaginal exposure in the treatment of post-traumatic stress disorder. Twelve-month follow-up. Br J Psychiatry 175:571-575.

Vasterling JJ, Brailey K, Constans JI, Sutker PB (1998) Attention and memory dysfunction in posttraumatic stress disorder. Neuropsychology 12:125-133.

Vouimba RM, Garcia R, Jaffard R (1998) Opposite effects of lateral septal LTP and lateral septal lesions on contextual fear conditioning in mice. Behav Neurosci 112:875-884.

Vouimba RM, Garcia R, Jaffard R (1999) Pretraining tetanic fimbrial stimulation impairs the expression but not the acquisition of contextual fear conditioning in mice. Neuroscience 93:869-876.

Vouimba RM, Garcia R, Baudry M, Thompson RF (2000) Potentiation of conditioned freezing following dorsomedial prefrontal cortex lesions does not interfere with fear reduction in mice. Behav Neurosci 114:720-724.

Yehuda R, McFarlane AC, Shalev AY (1998) Predicting the development of posttraumatic stress disorder from the acute response to a traumatic event. Biol Psychiatry 44:1305-1313. 